
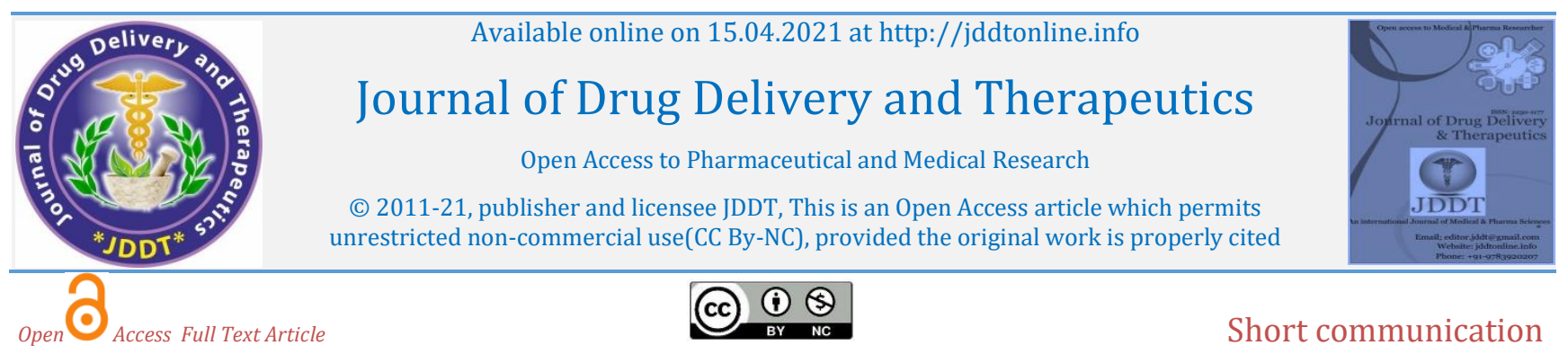

Short communication

\title{
In Silico Prediction and Pharmacokinetic Comparison of Ursodeoxycholic Acid and Obeticholic Acid in the Management of Primary Biliary Cholangitis
}

\author{
Manali Sudhir Dhage, Nila Ganamurali, Dhivya Dhanasekaran, Sarvesh Sabarathinam* \\ Department of Pharmacy Practice, SRM College of Pharmacy, SRM IST, Kattankulathur-603203, Tamil Nadu, India
}

\section{Article Info:

$\begin{array}{ll}\text { Article History: } & \\ & \text { Received 20 Feb 2021 } \\ \text { Accepted 07 April 2021 } & \text { Available online 15 April } 2021\end{array}$

Cite this article as:

Dhage MS, Ganamurali N, Dhanasekaran D, Sabarathinam S, In Silico Prediction and Pharmacokinetic Comparison of Ursodeoxycholic Acid and Obeticholic Acid in the Management of Primary Biliary Cholangitis, Journal of Drug Delivery and Therapeutics. 2021; 11(2-s):113-117 DOI: http://dx.doi.org/10.22270/jddt.v11i2-s.4669

\section{*Address for Correspondence:}

Sarvesh Sabarathinam, M Pharm., (Ph. D), Research scholar, Department of Pharmacy Practice, S.R.M. College of Pharmacy, SRM Institute of Science and Technology, SRM Nagar, Kattankulathur-603 203, Kancheepuram, Tamil Nadu, India.

\section{Abstract}

Background: Primary Biliary Cholangitis (PBC) is a persistent liver disease. Ursodeoxycholic acid is used as a first-line treatment for the past two decades. However, concurrent use of Ursodeoxycholic acid reported with a severe adverse drug reaction. Obeticholic acid has been started utilizing as monotherapy and also with Ursodeoxycholic acid in a patient who is intolerant to Ursodeoxycholic acid therapy. We primarily aimed to compare the pharmacokinetic \& toxicity profiles of Ursodeoxycholic acid and Obeticholic acid using in silico methods.

Method: The pharmacokinetic profile of UDCA \& OCA was observed from PKCSM server online database, OSIRIS ${ }^{\circledR}$ property Explorer, T.E.S.T. (Toxicity estimation software tool) \& Molinspiration ${ }^{\circledR}$ is used to estimate the drug toxicity profiles.

Result: This computer-aided response provides a great understanding and creates a gap between the theoretical and clinical evidence for UDCA \& OCA's preference in PBC management.

Conclusion: Co-administration of Obeticholic acid with Ursodeoxycholic acid will be an effective treatment for PBC in patients with UDCA intolerants. However, both medications are well-recognized substrates of the CYP3A4 enzyme and may lead to unintended drug interactions and side effects.

Keywords: Primary Biliary Cholangitis, Obeticholic acid, Ursodeoxycholic acid, CYP3A4, Drug Interactions, Pharmacokinetics.

\section{INTRODUCTION}

Primary Biliary Cholangitis (P.B.C.) is a persistent liver disease predominantly reported in middle-aged women, in a preponderant ratio of 8:1 (Female: Male). Patients with PBC are usually asymptomatic and are diagnosed via observation of serum alkaline phosphatase and or/total serum cholesterol. ${ }^{1}$ The incidence $\&$ prevalence rate of $\mathrm{PBC}$ ranges from 0.33 to 5.8 in 100,000 \& 1.91 to 40.2 in 100,000 patients respectively. ${ }^{2}$ The prevalence of P.B.C. heightened to around $100 /$ million in a year. ${ }^{3}$ Ursodeoxycholic acid (UDCA) has been utilized in the past 20 years as it impedes disease progression and improves survival rate without transplantation, so it has been recognized as a standard treatment option for P.B.C. The individual responsiveness of UDCA differs from one patient to another and shows an unfavourable worsening disease prognosis in unresponsive patients. Irrespective of the biochemical reaction, UDCA does not appear to cure the cardinal symptoms of PBC 4 . Evidence from early clinical trials shows UDCA produces significant improvement in bilirubin levels and prevents the development of the disease. An analysis of three major clinical studies showed that daily doses of $13-15 \mathrm{mg} / \mathrm{kg}$ of UDCA for a period of 4 years has a tendency to reduce the need for a liver transplant. ${ }^{5}$ UDCA also has the potential to function as a hepatoprotective agent, but hepatocellular carcinoma has been reported when biochemical response not achieved $9 \%$ in 10 years and $20 \%$ in 15years. UDCA produces unintended toxicities such as pruritus, hepatitis, cholangitis, vanishing bile duct syndrome, immunesuppression liver cell failure, ascites, extremely watery diarrhoea, pneumonia, dysuria \& mutagenic consequences. ${ }^{6}$ The primary purpose of this narrative review is to discuss the pharmacokinetic correlations and in-silico toxicity profiles of UDCA \& Obeticholic acid (OCA) in the treatment of $\mathrm{PBC}$ and its clinical outcome.

\subsection{Overview of OCA}

In the year 2016, OCA was approved \& recommended for the treatment of P.B.C. in combination with UDCA when insufficient feedback was reported after UDCA monotherapy. It is used as monotherapy in P.B.C. patients who are intolerant to UDCA. OCA is an effective and dynamic agonist of the Farnesoid X Receptor (F.X.R.). This receptor belongs to the nuclear receptor family, which is primarily involved in bile acid synthesis and transportation and is present in the liver and small intestine. Apart from F.X.R., OCA is also responsible for activating the G-protein coupled receptor GPBAR1/TGR5 for secondary bile acids. OCA is 
approximately 100 times more potent than its parent compound, chenodeoxycholic acid, which makes it an ideal drug for the treatment of several hepatic disorders. The proposed mechanism of action of OCA is said to be that upon binding to F.X.R., gene transcription of the enzyme cholesterol $7 \alpha$-hydroxylase is inhibited by induction of Small Heterodimer Protein (S.H.P.) in liver and Fibroblast Growth Factor-19 (FGF-19) in the small intestine. This is a significant enzyme involved in the conversion of cholesterol to bile acids. Furthermore, the expression of the Bile Salt Excretory
Pump (B.S.E.P.), which is responsible for the efflux of bile salts, is also increased. The recommended initial therapy of OCA is 5 mg once weekly for patients who are categorized as Class B and C in the Child-Pugh assessment. 7-9 The drug dose has been titrated to $10 \mathrm{mg}$, especially for those who have not achieved the anticipated reduction of ALP and or total bilirubin in the first three months, and $10 \mathrm{mg}$ is considered as the maximum dose. Pruritus and fatigue are the most frequently documented adverse drug reactions. The pharmacokinetic profile of OCA is given in Table 1.

Table 1: Pharmacokinetic assessment of UDCA\&OCA

\begin{tabular}{|c|c|c|}
\hline Parameters & UDCA & OCA \\
\hline Water solubility (log mol/L) & -4.19 & -4.268 \\
\hline CaCo2 permeability $\left(\log\right.$ Papp in $\left.10^{-6} \mathrm{~cm} / \mathrm{s}\right)$ & 0.705 & 0.684 \\
\hline Intestinal absorption (\% Absorbed) & 95.75 & 96.678 \\
\hline Skin Permeability (log Kp) & -2.733 & -2.733 \\
\hline VDss(Human) (log L/kg) & -0.88 & -1.036 \\
\hline Fraction Unbound (Fu) & 0.063 & 0.03 \\
\hline Substrate & CYP3A4 & CYP3A4 \\
\hline Total clearance $(\log \mathrm{ml} / \mathrm{min} / \mathrm{kg})$ & 0.607 & 0.624 \\
\hline AMES Toxicity & NO & NO \\
\hline Oral route Acute Toxicity (LD50) (mol/kg) & 2.659 & 2.613 \\
\hline Oral route chronic toxicity & 1.853 & 2.221 \\
\hline \multicolumn{3}{|l|}{ (log mg/kg_bw/day) } \\
\hline Hepatotoxicity & NO & NO \\
\hline Skin sensitization & NO & NO \\
\hline Minnow toxicity $(\log \mathrm{mM})$ & 1.166 & -0.263 \\
\hline
\end{tabular}

\subsection{Overview of UDCA:}

UDCA is indicated by US FDA for the management of patients with PBC UDCA is a normal constituent of human bile. The majority of the UDCA is presented in a conjugated form with glycine. ${ }^{10}$ Numerous mechanisms of actions of UDCA in the pathology of liver diseases have been implicated, but these vary according to the stage of the cholestatic disease. In the primitive stages of Primary Biliary Cholangitis (PBC), the major mechanism of UDCA action is safeguarding injured hepatic cells against noxious effects of bile acids. Also, the activity and number of transporter proteins in the canalicular membrane determine the secretion capacity of cholangiocytes. In the case of drug-induced hepatic disease, transporter function may be pathologically impaired, but UDCA stimulates biliary secretion. Chemical structures of UDCA \& OCA given in Figure 1. 11,12

\section{METHODS}

A broad literature review was performed to compile the mechanisms of action of the targeted drugs such as "Ursodeoxycholic acid" and "Obeticholic acid" The pharmacokinetic profile of UDCA \& OCA was observed from the PKCSM online server. ${ }^{13}$ Toxicity prediction \& druglikeness was performed using OSIRIS Property Explorer program and Molinspiration software.

\subsection{In silico prediction for toxicity:}

Toxicity is accountable for the withdrawal and failure of new chemical entities. The toxicity profile of selected drugs was analyzed through the OSIRIS $₫$ Property Explorer program. This tool is accessible through cheminformatics.ch and chemistry.org. It is a freely available online software program that forecasts potential side effects such as mutagenicity, tumorigenicity, irritant, reproductive effects, drug-likeness and physicochemical properties analogous with a compound in a colour-coded format. The green colour indicates drug conform behaviour, yellow indicates medium risk, whereas the red colour shows a high risk for mutagenicity or low intestinal absorption. (Table 2) These predictions are essential to prevent deleterious substances to advance in drug discovery and development. We have also compared certain drug-related parameters such as Topological Polar Surface Area (T.P.S.A.), drug-likeness and overall drug score. ${ }^{14-16}$

(a) T.P.S.A. - Blood-brain barrier penetration and intestinal absorption are bioavailability-associated properties that are well correlated with T.P.S.A. and is calculated as the total sum of the contribution of fragments, mainly $\mathrm{O}^{-}$and $\mathrm{N}^{-}$ fragments are considered.

(b) Drug likeness- A fragment-based approach is used for estimating drug-likeness via OSIRIS program. A positive value demonstrates that the study compound contains those 
fragments which are found in commercially available formulations.

(c) Overall drug score - This is calculated using criterions such as drug-likeness, molecular weight, toxicity risk, log $\mathrm{S}$ and $\log \mathrm{P}$ values. A score of $>0.5$ along with minimal toxicity risk is considered favorable.

Table 2: Toxicity profile of OCA and UCA as predicted via OSIRIS Property Explorer program.

\begin{tabular}{|l|l|l|}
\hline PARAMETERS & $\begin{array}{l}\text { Obeticholic acid } \\
\text { (scores) }\end{array}$ & $\begin{array}{l}\text { Ursodeoxycholic acid } \\
\text { (scores) }\end{array}$ \\
\hline Mutagenic & Green & Green \\
\hline Tumorigenic & Green & Green \\
\hline Irritant & Green & Green \\
\hline Reproductive effect & Green & Green \\
\hline TPSA & 60.69 & 77.76 \\
\hline Drug likeness & -1.96 & -0.41 \\
\hline Drug score & 0.28 & 0.51 \\
\hline
\end{tabular}

\section{Toxicity predictions}

T.E.S.T. (Toxicity estimation software tool) ${ }^{\circledR}$ Version 5.1 is used to calculate the toxicity profile of selected bioactive compounds. This system generally comprises a receptor essential amino acids enzyme histidine kinase (H.K.) that will react to an extracellular signal by phosphorylating cytoplasmic response regulator. ${ }^{17}$ [Table 3]

Table 3: Toxicity estimation of selected bioactive compounds using T.E.S.T software

\begin{tabular}{|l|l|l|}
\hline \multicolumn{1}{|c|}{ Parameters } & \multicolumn{1}{|c|}{ Obeticholic acid } & \multicolumn{1}{c|}{ Ursodeoxycholic acid } \\
\hline $\begin{array}{l}\text { Fathead minnow LC50 }(96 \mathrm{hr})- \\
\text { Log10(mol/L) }\end{array}$ & 5.87 & 1.55 \\
\hline $\begin{array}{l}\text { Fathead minnow LC50 }(96 \mathrm{hr}) \\
\text { mg/L }\end{array}$ & 0.56 & 0.88 \\
\hline $\begin{array}{l}\text { Similarity coefficient } \\
\text { ( } \geq 0.5)\end{array}$ & 0.81 & 4.63 \\
\hline $\begin{array}{l}\text { Daphnia magna LC50 }(48 \mathrm{hr})- \\
\text { Log10(mol/L) }\end{array}$ & 4.80 & 9.27 \\
\hline $\begin{array}{l}\text { Daphnia magna LC50 (48 hr) } \\
\text { mg/L }\end{array}$ & 6.66 & 0.61 \\
\hline $\begin{array}{l}\text { Similarity coefficient } \\
\text { ( } \geq 0.5)\end{array}$ & 0.61 & 1.33 \\
\hline Bioconcentration factor Log10 & 1.45 & 21.58 \\
\hline Bioconcentration factor & 28.38 & 0.83 \\
\hline $\begin{array}{l}\text { Similarity coefficient } \\
\text { ( } \geq 0.5)\end{array}$ & 0.83 & \\
\hline
\end{tabular}

Similarity coefficient $(\geq 0.5$ ) comparing the category of similar structures form the existing compounds.

\section{Molecular property:}

The designed and docked molecules were screened in silico using MOLINSPIRATION $®$ software to evaluate the druglikeness of the compounds. This software is also equipped with data visualization, bioactivity prediction and fragmentbased virtual screening. Any molecule possessing a bioactivity score of more than 0.00 is said to have a favourable biological activity, whereas a score of -0.50 to 0.00 renders a molecule to contain moderate activity, and a score $<0.00$ is said to inactive. Those molecules with the highest score are likely to be more active. 18-20. Four important drug-receptor classes are screened - G-Protein Coupled Receptor ligands (G.P.C.R.), nuclear receptor ligands, tyrosine kinase inhibitors, ion channel inhibitors. (Table 4) 
Table 4: Evaluation of bioactivity scores gathered from Molinspiration Database.

\begin{tabular}{|c|c|c|}
\hline parameters & $\begin{array}{c}\text { Obeticholic acid } \\
\text { (Scores) }\end{array}$ & $\begin{array}{c}\text { Ursodeoxycholic acid } \\
\text { (Scores) }\end{array}$ \\
\hline GPCR ligand & 0.24 & 0.33 \\
\hline Ion channel modulator & 0.13 & 0.33 \\
\hline Kinase inhibitor & -0.48 & 0.39 \\
\hline Nuclear receptor ligand & 0.80 & 0.86 \\
\hline Protease inhibitor & 0.20 & 0.68 \\
\hline Enzyme inhibitor & 0.61 & 0.29 \\
\hline
\end{tabular}

\section{RESULTS}

UDCA is used as first-line therapy in the management of biliary cholangitis. However, patients who were treated with UDCA reported severe adverse drug reaction. This theoretical computer-aided response offers a great understanding of the preference of UDCA \& OCA in the management of PBC. The pharmacokinetic parameters of both medications did not indicate dramatic differences. Yet, the half-life of UDCA is 3.5-5.8 days, whereas the half-life of OCA is 24 hours. The intestinal absorption of UDCA is $95.75 \%$, and OCA is approximately $96.678 \%$. Total clearance $(\log \mathrm{ml} / \mathrm{min} / \mathrm{kg})$ of UDCA \& OCA were $0.607 \& 0.624$ respectively. A.M.E.S. Toxicity\& hepatotoxicity have not been reported for both drugs. After the full phase of metabolism, both of the drugs are excreted through faeces.

Both OCA and UDCA do not show mutagenic or tumorigenic responses, respectively. However, the overall drug score was lesser than UDCA. OCA was found to have a strong biological activity at nuclear receptor ligand and enzyme inhibitor, moderate activity at G.P.C.R., ion channel receptor and protease inhibitor. Its activity at the kinase receptor was found to be negligible.

\section{DISCUSSION:}

Even in the absence of serious liver disorders, P.B.C. is strongly associated with morbidity \& mortality. During the drug metabolism process, CYP3A4 contributes to a major extent for the metabolism of $65 \%$ xenobiotics, with UDCA and OCA being substrates of it.

UDCA is used as a first-line treatment for P.B.C., which improves liver function and reduces the development of hepatic fibrosis, the formation of oesophagal varices as well as delaying the need for liver transplantation. About onethird of patients do not respond to UDCA therapy, i.e. 30\% of western patients and $32 \%-44.7 \%$ of Chinese patients failed to give an adequate response to UDCA therapy. ${ }^{21}$ Ageing is a patient-specific factor that is not largely considered but can also give rise to unintended interactions due to changes in systemic absorption with advancing age. A comparative study of P.K. parameters of UDCA among healthy, elderly volunteers and younger adults showed that UDCA conjugate ratio and rate of biotransformation was lower in elderly adults when compared with younger adults. ${ }^{22}$

A 6-week, 25 or $50 \mathrm{mg}$ administration of OCA improves the insulin sensitivity \& reduces liver inflammation in adults with type 2 diabetes mellitus and nonalcoholic fatty disorder of the liver, respectively. ${ }^{23}$ Pruritus was one of the major reported adverse drug reactions of OCA However, a significant difference in the incidence of pruritus cases is based on the dose given. ${ }^{24}$ Intake of food may increase the absorption of OCA as exhibited in a study wherein the $\mathrm{AUC}_{0-\mathrm{t}}$ of test drug and $\mathrm{AUC}_{0-\infty}, \mathrm{AUC}_{0-\mathrm{t}}$ of reference product under fed condition was found to be higher than fasting condition.

As the half-lives of these two drugs overlap, concurrent administrations of these CYP3A4 substrates may have possibilities of interfering with the CY3A4 isoenzyme, leading to a severe adverse drug reaction or therapeutic failure. However, a phase-1 study assessing drug interactions between OCA and digoxin, warfarin, midazolam, caffeine, dextromethorphan, omeprazole and rosuvastatin reported that there was no significant inhibition of P.K. parameters of dextromethorphan, S-warfarin and digoxin at both $10 \mathrm{mg}$ and $25 \mathrm{mg}$ doses. P.K. of rosuvastatin was moderately suppressed with the $25 \mathrm{mg}$ dose as an increase in plasma concentration was observed, and a weak interaction was found between OCA and caffeine.

\section{CONCLUSION:}

We believe that the co-administration of Obeticholic acid with Ursodeoxycholic acid would be an effective treatment for P.B.C. in patients with UDCA intolerance. In order to avoid severe adverse drug reaction/ therapeutic failure, extra care and monitoring are recommended when concomitant administration of UDCA+OCA is warranted.

\section{Ethics Approval and Consent to Participate}

Not applicable

\section{Human and Animal Rights}

No animals/humans were used for studies that are base of this research.

\section{Consent for Publication}

Not applicable.

\section{Funding}

None

\section{Conflict of Interest}

The authors declare no conflict of interest, financial or otherwise.

\section{Availability of Data and Materials}

Not appliance.

\section{Acknowledgements}

We would like to thank all health care professionals. 


\section{REFERENCES}

1. Kumagi T, Heathcote E. Primary biliary cirrhosis. Orphanet J Rare Dis 2008; 3:1.

2. Marzioni M, Bassanelli C, Ripellino C, Urbinati D, Alvaro D, Epidemiology of primary biliary cholangitis in Italy: Evidence from a real-world database. Dig Liver Dis 2019; 51(5):724-729.

3. Borman M, Swain MG. Changing epidemiology and natural history of primary biliary cirrhosis. Clin Liver Dis (Hoboken) 2014; 10,3(1):12-14.

4. Marschall HU, Henriksson I, Lindberg S, Söderdahl F, Thuresson M, Wahlin S, Ludvigsson, JF. Incidence, prevalence, and outcome of primary biliary cholangitis in a nationwide Swedish population-based cohort. Sci Rep 2019, 8,9(1):11525.

5. Paumgartner G. Ursodeoxycholic acid for primary biliary cirrhosis: treat early to slow progression. J Hepatol 2003; 39(1):112-4.

6. Kotb M.A. Molecular mechanisms of ursodeoxycholic acid toxicity \& side effects: ursodeoxycholic acid freezes regeneration \& induces hibernation mode. Int J Mol Sci 2012; 13(7):8882-914.

7. Fiorucci S, Di Giorgio C, Distrutti E. Obeticholic Acid: An Update of Its Pharmacological Activities in Liver Disorders. Handb Exp Pharmacol 2019; 256:283-295.

8. Modica S, Petruzzelli M, Bellafante E, et al. Selective activation of nuclear bile acid receptor F.X.R. in the intestine protects mice against cholestasis. Gastroenterology 2012; 142(2):355-65.

9. Shah RA, Kowdley KV. Current and potential treatments for primary biliary cholangitis. Lancet Gastroenterol Hepatol 2020 5(3):306-315.

10. Cabrera D, Arab JP, Arrese M. UDCA, NorUDCA, and T.U.D.C.A. in Liver Diseases: A Review of Their Mechanisms of Action and Clinical Applications. Handb Exp Pharmacol 2019; 256:237264.

11. Ikegami T, Matsuzaki Y. Ursodeoxycholic acid: Mechanism of action and novel clinical applications. Hepatol Res 2008; 38(2):123-131.

12. Paumgartner G, Beuers U. Mechanisms of action and therapeutic efficacy ofursodeoxycholic acid in cholestatic liver disease. Clin Liver Dis 2004; 8(1):67-vi.

13. Pires DE, Blundell TL, Ascher DB. pkCSM: Predicting SmallMolecule Pharmacokinetic and Toxicity Properties Using Graph-
Based Signatures. Journal of medicinal chemistry 2015; 58(9):4066-4072.

14. Ayati A, Falahati M, Irannejad H, Emami S. Synthesis, in vitro antifungal evaluation and in silico study of 3-azolyl-4chromanone phenylhydrazones. Daru 2012; 20(1):46.

15. Rashid M. Design, synthesis and ADMET prediction of bis benzimidazole as anticancer agent. Bioorg Chem 2020; 96:103576.

16. Osman W, Ismail E.M.O.A, Shantier SW, Mohammed MS, Mothana RA, Muddathir A, Khalid HS. In silico assessment of potential leads identified from Bauhinia rufescens Lam. as $\alpha$ glucosidase and $\alpha$-amylase inhibitors. J Recept Signal Transduct Res 2021; 41(2):159-169.

17. Martin T, P. Harten, D. Young. TEST (Toxicity Estimation Software Tool) Ver 4.1. U.S. Environmental Protection Agency, Washington, DC, E.P.A./600/C-12/006, 2012.

18. Husain A, Ahmad A, Khan SA, Asif M, Bhutani R, Al-Abbasi FA. Synthesis, molecular properties, toxicity and biological evaluation of some new substituted imidazolidine derivatives in search of potent anti-inflammatory agents. Saudi Pharm J 2016; 24(1):104-14.

19. Kumar N, Mishra SS, Sharma CS, Singh HP, Pandiya H. In silico Pharmacokinetic, Bioactivity and Toxicity Evaluation of Some Selected Anti-Ulcer Agents. Int J Pharm Sci Res 2017; 9(2):6871.

20. Hassan M, Ashraf Z, Abbas Q, Raza H, Seo SY. Exploration of Novel Human Tyrosinase Inhibitors by Molecular Modeling, Docking and Simulation Studies. Interdiscip Sci 2018; 10(1):6880.

21. Laschtowitz A, de Veer RC, Van der Meer AJ, Schramm C. Diagnosis and treatment of primary biliary cholangitis. United European Gastroenterol J 2020; 8(6):667-674.

22. Mudaliar S, et al. Efficacy and safety of the farnesoid X receptor agonist obeticholic acid in patients with type 2 diabetes and nonalcoholic fatty liver disease. Gastroenterology 2013; 145(3):574-82.

23. Nevens F, et al. POISE Study Group. A Placebo-Controlled Trial of Obeticholic Acid in Primary Biliary Cholangitis. N Engl J Med 2016; 375(7):631-43.

24. Sabarathinam S, Vijayakumar TM, A short exploration of selected sensitive CYP3A4 substrates (Probe Drug). Drug Metabolism Letters, 2020, 14:1. 